\title{
The concurrent validity and reliability of the Leg Motion system for measuring ankle dorsiflexion range of motion in older adults
}

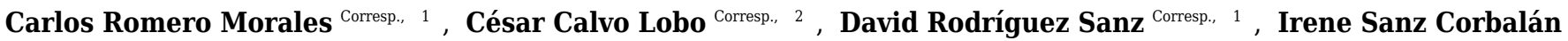 \\ Corresp., ${ }^{3}$, Beatriz Beatriz Ruiz Ruiz ${ }^{1}$ ， Daniel López López ${ }^{\text {Corresp. } 4}$ \\ 1 Physiotherapy Department, Faculty of Health, Exercise and Sport, Universidad Europea de Madrid, Villaviciosa de Odón, Madrid, España \\ 2 Departamento de Fisioterapia. Centro Superior de Estudios Universitarios La Salle. Universidad Autónoma de Madrid. Spain. Motion in Brains Research \\ Group. Instituto de Neurociencias y Ciencias del Movimiento. Centro Superior de Estudios Universitarios La, Universidad Autónoma de Madrid, Madrid, \\ Madrid, España \\ 3 School of Nursing, Physiotherapy and Podiatry, Universidad Complutense de Madrid, Madrid, Madrid, España \\ 4 Research, Health and Podiatry Unit. Department of Health Sciences. Faculty of Nursing and Podiatry, Universidade da Coruña, Ferrol, A Coruña, Spain \\ Corresponding Authors: Carlos Romero Morales, César Calvo Lobo, David Rodríguez Sanz, Irene Sanz Corbalán, Daniel López López \\ Email address: carlos.romero@universidadeuropea.es, cecalvo19@hotmail.com, davidrodriguezsanz@gmail.com, iresanzcorbalan@gmail.com, \\ daniellopez@udc.es
}

Background: New reliable devices about range of motion (ROM) measures in older adults are necessary to improve knowledge about functional capability in this population. Dorsiflexion ROM limitation is associated with ankle injuries, foot pain, lower limb disorders, loss of balance, gait control disorders and fall risk in older adults. The aim of the present study was to assess the validity and reliability of the Leg Motion device for measuring ankle dorsiflexion ROM in older adults. Methods: A descriptive repeatedmeasures study was designed to test the reliability of Leg Motion in thirty-three healthy elderly patients older than 65 years. The subjects had to meet the following inclusion and exclusion criteria in their medical records: older than 65 years; no lower extremity injury for at least one year prior to evaluation (meniscopathy, or fractures) and any chronic injuries (e.g., osteoarthritis); no previous hip, knee or ankle surgery; no neuropathic alterations and no cognitive conditions (e.g., Alzheimer's disease or dementia). Participants were recruited through the person responsible for the physiotherapist area from a nursing center. The subjects were evaluated in two different sessions at the same time of day, and there was a break of two weeks between sessions. To test the validity of the Leg Motion system, the participants were measured in a weight-bearing lunge position using a classic goniometer with $1^{\circ}$ increments (Baseline, USA), a smartphone with an inclinometer standard app (iPhone $5 \mathrm{~S}^{\circledR}$ ) with $1^{\circ}$ increments and a measuring tape that could measure $0.1 \mathrm{~cm}$. All testing was performed while the patients were barefoot. The researcher had ten years of experience as a physiotherapist using goniometer, tape measure and inclinometer devices. Results: Mean values and standard deviations were as 
follows: Leg Motion (right $5.15 \pm 3.08$; left $5.19 \pm 2.98$ ), tape measure (right $5.12 \pm 3.08$; left $5.12 \pm 2.80$ ), goniometer (right $45.87^{\circ} \pm 4.98$; left $44.50^{\circ} \pm 5.54$ ) and inclinometer app (right $46.53^{\circ} \pm 4.79$; left $45.27^{\circ} \pm 5.19$ ). The paired t-test showed no significant differences between the limbs or between the test and re-test values. The test re-test reliability results for Leg Motion were as follows: the standard error of the measurement ranged from 0.29 to $0.43 \mathrm{~cm}$, the minimal detectable difference ranged from 0.79 to 1.19 $\mathrm{cm}$, and the intraclass correlation coefficients (ICC) values ranged from 0.97 to 0.98 . Conclusions: The results of the present study indicated that the Leg Motion device is a valid, reliable, accessible and portable tool as an alternative to the classic weight-bearing lunge test for measuring ankle dorsiflexion ROM in older adults. 


\section{Research paper}

The concurrent validity and reliability of the Leg Motion system for measuring ankle dorsiflexion range of motion in older adults

2

3 Authors:

4

5 Carlos Romero Morales, PhD, Msc, PT (1), carlos.romero@universidadeuropea.es

6 César Calvo Lobo, PhD, Msc, PT (2), cecalvo19@hotmail.com

7 David Rodríguez Sanz, PhD, MsC, PT, DP (1), davidrodriguezsanz@gmail.com

8 Irene Sanz Corbalan, PhD, MSc, DP (3), iresanzcorbalan@gmail.com

9 Beatriz Ruiz Ruiz, PhD, Msc, PT (1), beatriz.ruiz@universidadeuropea.es

10 Daniel López López, PhD, BSc, MSc, DP (4) daniellopez@udc.es

11

12 (1) Lecturer, Physiotherapy Department, Faculty of Health, Exercise and Sport, European 13 University of Madrid, Villaviciosa de Odón, Madrid, Spain.

14 (2) Physiotherapy Department, Motion in Brains Research Group, Instituto de Neurociencias y 15 Ciencias del Movimiento, Centro Superior de Estudios Universitarios La Salle, Universidad 16 Autónoma de Madrid, Spain.

17 (3) School of Nursing, Physiotherapy and Podiatry. Universidad Complutense de Madrid, Spain.

18 (4) Research, Health and Podiatry Unit. Department of Health Sciences. Faculty of Nursing and 19 Podiatry. Universidade da Coruña, Spain. 


\section{Address correspondence and reprint requests to:}

24 Daniel López López

25 Universidade da Coruña

26 Unidade de Investigación Saúde e Podoloxía

27 Departamento de Ciencias da Saúde

28 Campus Universitario de Esteiro s/n

2915403 Ferrol (España)

30 Email: daniellopez@udc.es 


\section{Research paper}

\section{The concurrent validity and reliability of the Leg Motion system for measuring ankle dorsiflexion range of motion in older adults}

\section{ABSTRACT}

34 Background: New reliable devices about range of motion (ROM) measures in older adults are necessary to improve knowledge about functional capability in this population. Dorsiflexion ROM limitation is associated with ankle injuries, foot pain, lower limb disorders, loss of balance, gait disorders and fall risk in older adults. The aim of the present study was to assess the validity and reliability of the Leg Motion device for measuring ankle dorsiflexion ROM in older adults. Methods: A descriptive repeated-measures study was designed to test the reliability of Leg Motion in thirty-three healthy elderly patients older than 65 years. The subjects had to meet the following inclusion and exclusion criteria in their medical records: older than 65 years; no lower extremity injury for at least one year prior to evaluation (meniscopathy, or fractures) and any chronic injuries (e.g., osteoarthritis); no previous hip, knee or ankle surgery; no neuropathic alterations and no cognitive conditions (e.g., Alzheimer's disease or dementia). Participants were recruited through the person responsible for the physiotherapist area from a nursing center. The subjects were evaluated in two different sessions at the same time of day, and there was a break of two weeks between sessions. To test the validity of the Leg Motion system, the participants were measured in a weight-bearing lunge position using a classic goniometer with $1^{\circ}$ increments (Baseline, USA), a smartphone with an inclinometer standard app (iPhone $5 \mathrm{~S}^{\circledR}$ ) with $1^{\circ}$ increments and a measuring tape that could measure $0.1 \mathrm{~cm}$. All testing was performed while the patients were barefoot. The 
52 inclinometer devices. Results: Mean values and standard deviations were as follows: Leg Motion 53 (right 5.15 \pm 3.08 ; left 5.19 \pm 2.98 ), tape measure (right 5.12 \pm 3.08 ; left $5.12 \pm 2.80$ ), goniometer 54 (right $45.87^{\circ} \pm 4.98$; left $\left.44.50^{\circ} \pm 5.54\right)$ and inclinometer app (right $46.53^{\circ} \pm 4.79$; left $45.27^{\circ} \pm$ 55 5.19). The paired t-test showed no significant differences between the limbs or between the test 56 and re-test values. The test re-test reliability results for Leg Motion were as follows: the standard 57 error of the measurement ranged from 0.29 to $0.43 \mathrm{~cm}$, the minimal detectable difference ranged 58 from 0.79 to $1.19 \mathrm{~cm}$, and the intraclass correlation coefficients (ICC) values ranged from 0.97 to 59 0.98. Conclusions: The results of the present study indicated that the Leg Motion device is a valid, 60 reliable, accessible and portable tool as an alternative to the classic weight-bearing lunge test for 61 measuring ankle dorsiflexion ROM in older adults.

62 


\section{INTRODUCTION}

65 Range of motion (ROM) measurements are considered to be an important factor in the 66 physiotherapeutic assessment of the general population and of special groups, such as sportsmen ${ }^{1}$ 67 and older adults. ${ }^{2}$ Changes in ankle dorsiflexion ROM have been associated with foot pain, ankle 68 injuries, ${ }^{3}$ neuritis and lower limb disorders. ${ }^{4}$

69 Spain tops the list of the European countries that require a higher rate of geriatric care, and is a 70 clear example of growing elderly population. ${ }^{5}$

71 It is known that the aging process in elderly people reduces quality of life and causes modifications

72 in the locomotor system, ${ }^{6}$ reducing the ROM and limiting mobility. A restricted ROM may 73 predispose individuals to a lack of coordination ${ }^{7}$, can increase the fall risk $^{8}$, is an excellent 74 predictor of the loss of ambulation in elderly people ${ }^{9}$ and it is presented in many adult pathologies 75 that affect postural control and gait. Daily activities such as walking, descending stairs, and 76 kneeling require $10^{\circ}$ of ankle dorsiflexion $\mathrm{ROM}{ }^{10}$, while other actions such as running require $20^{\circ}$ 77 to $30^{\circ} \cdot{ }^{11}$

78 It is very common in the literature to find studies, whose objective is to validate measurement 79 instruments for the elderly population. ${ }^{12,13}$

80 In the study of Slavko et al., ${ }^{12}$ it is stated that there are several methods to assess gait in humans, 81 but its feasibility has not been demonstrated in frail populations. In the case of ROM 82 measurements, there are several studies that attempt to validate the applicability of different 83 instruments for elderly adults, as reported Sacco and colleagues which aims to validate a measuring 84 instrument in the elbow joint range in gerontology, ${ }^{2}$ although no studies have been found for ankle 85 dorsiflexion. 
86 Based on a reliability dorsiflexion ROM study, the weight-bearing measures are more reliable 87 (ICC $=0.93-0.96)$ than non-weight-bearing postures $(\mathrm{ICC}=0.32-0.72)$ during practical activities, 88 such as walking, running or stair ambulation. ${ }^{14}$ Multiple tools, such the inclinometer, goniometer 89 and tape measure, have been used to measure the ankle ROM. ${ }^{15}$

90 Tape measure is an easy way to determine ankle flexion ROM in the weight bearing position. ${ }^{14}$ 91 According to Bennell et al. ${ }^{16}$ the test starts in the standing position, with the subject's foot on the 92 tape line, perpendicular to the wall. The subject then moves the foot away from the wall until the 93 knee touches the wall lightly without lifting the heel from the ground. The distance between the 94 big toe and the wall (in centimeters) is then measured. A tape measure is a cheap tool that can 95 easily be transported and quickly and safely used in many settings. But there are some potential 96 variations that occur during testing that need to be controlled. For instance, variations in the 97 subtalar and foot position ${ }^{17}$ and the visual reference for the knee or the maintenance of the foot 98 alignment during the performance of the test may change dorsiflexion results ${ }^{18}$ are the main 99 limitations with regard to the standardization of this test.

100 The goniometer is frequently used to evaluate ankle joint dorsiflexion. ${ }^{18}$ It is an inexpensive and 101 portable tool, but experience is required for its accuracy and effective use.

102 Another approach to quantifying the ankle dorsiflexion ROM is an inclinometer; Venturini et al. ${ }^{19}$ 103 reported reliable results when performing measurements with a digital inclinometer (ICC $=0.84$ $1040.95)$ compared with a goniometer $(\mathrm{ICC}=0.65-0.89)$. Smartphones are accessible and easy to 105 handle devices, with inclinometer apps. ${ }^{20}$ Wellmon et al. ${ }^{21}$ performed a validity and reliability 106 examination of three different smartphones inclinometer apps (iPhone $5^{\circledR}$, Samsung SII and LG). 107 The interrater reliability ( $\mathrm{ICC}=0.995-1.000)$ and validity $(\mathrm{ICC}=0.998-0.999)$ were excellent. It is 108 only necessary for the implementation of this measurement, finding the tibial tuberosity for 
109 stabilizing the smartphone in a weight-bearing position. The inclinometer uses a digital display to

110 report the angle of decline. ${ }^{22}$ Despite the high intraclass correlation coefficient values for the

111 goniometer and inclinometer, there is no universal agreement on the choice of one method over

112 the other. ${ }^{15}$

113 Check your MOtion (Spain) developed the Leg Motion system, a new, accessible, lightweight and

114 portable tool for evaluating the ankle dorsiflexion ROM in the weight-bearing position. ${ }^{23}$ During 115 assessment with the Leg Motion system, the big toe is placed at the starting line and the knee

116 touches a metal stick while keeping the foot in the same position without removing the heel from

117 the surface. The metal stick is progressed along the line to the maximal ankle dorsiflexion. Leg

118 Motion shows greater standardization because can be applied on any surface and doesn't need the

119 presence of a wall during the measurement. Therefore, Leg Motion is considered to be a portable

120 tool that can be tested in any location or on any surface, "where a measuring tape needs to be

121 placed or where the normal weight-bearing lunge test has limitations, for example: the variations

122 in the subtalar and foot position during the measurement," according to Calatayud et al., ${ }^{23}$ who

123 provided evidence to support the use of the Leg Motion device in healthy subjects.

124 The aim of the present study was to test the validity and reliability of Leg Motion for measuring 125 ankle dorsiflexion ROM in older adults.

126

\section{METHODS}

129 Study design

130 A descriptive repeated-measures study was performed between April and June 2015.

\section{Participants}


132 Thirty-three healthy elderly subjects (age $71 \pm 3.6$ years, height $167.0 \pm 10 \mathrm{~cm}$, weight $68.24 \pm$

$13313.47 \mathrm{~kg}$, and body mass index $24.31 \pm 3.50 \mathrm{~kg} / \mathrm{m}^{2}$ ) were included in the study. Before starting the

134 procedure, all the participants read and signed an institutional informed consent. All protocols used

135 in the study comply with the items listed in the 1975 Declaration of Helsinki and its 2008 review.

136 Participants were recruited through the person who was responsible for the physiotherapist area at

137 a care center. The subjects had to meet the following inclusion and exclusion criteria: over 65 years

138 of age; no lower extremity injury for at least one year prior to the evaluation (i.e., meniscopathy,

139 fractures), and any chronic injuries (i.e., osteoarthritis); no previous hip, knee or ankle surgery; no

140 neuropathic alterations and no cognitive conditions (i.e., Alzheimer's disease and dementia). The

141 scientific committee of European University of Madrid (CIPI/048/15) approved this study.

142

143 Procedure

144 In accord with the protocol established by Calatayud et al. ${ }^{23}$ the subjects were evaluated in two

145 different sessions at the same time of day and there was a break of two weeks between sessions.

146 The participants were measured in a weight-bearing lunge position using a classic goniometer with

$1471^{\circ}$ increments (Baseline, USA), a smartphone with an inclinometer standard app (iPhone $5 \mathrm{~S}^{\circledR}$ ) with

$1481^{\circ}$ increments and a measuring tape that could measure $0.1 \mathrm{~cm}$. All testing was performed while

149 the patients were barefoot and three trials were performed for each leg, in each testing method.

150 The researcher had ten years of experience as a physiotherapist and using a goniometer, tape

151 measure and inclinometer.

152 Leg Motion is a new device; therefore the physiotherapist had only one and a half years' experience

153 with this tool. 
154 The tape measure protocol was performed with the participants in the standing position with their

155 heels touching the ground, knees aligned with the second toe, and the big toe $10 \mathrm{~cm}$ away from the 156 wall (Figure 1).

159 The participants could be lightly supported on the wall using the index and middle fingers of each

160 hand. As proposed by Konor et al. ${ }^{15}$ the subjects were encouraged to move their knees toward the

161 wall (maintaining alignment with the second toe) until their knees touched the wall. The foot

162 continued to move away from the wall $1 \mathrm{~cm}$ at a time, and the participants performed this exercise

163 again until they were not able to contact the wall with their knees without lifting their heels off the

164 surface. From that point on, the foot moved to the nearest $0.1 \mathrm{~cm}$ increments away from the

165 baseline until the knee contacted the wall. ${ }^{24}$

166 Maximal dorsiflexion ROM during measuring tape test was defined as the maximum distance of

167 the toe from the wall, while maintaining contact between the wall and knee, without lifting the

168 heel. ${ }^{15}$ This thorough procedure was performed to ensure accuracy to the nearest millimeter. ${ }^{24}$

169 Once the patient reached the final lunge position at maximal dorsiflexion, a classic goniometer 170 was aligned with the fibula (mobile branch) and the floor (stable branch) (Figure 2). ${ }^{15}$

173 After the goniometer measurement, in the same position, an iPhone $5 \mathrm{~S}^{\circledR}$ with an inclinometer app 174 was placed at the tibial tuberosity to evaluate the angle between the tibia and the ground (Figure $1753) \cdot{ }^{15}$ 
178 Finally, according to the procedure by Calatayud et al. ${ }^{23}$ for the Leg Motion device, patients were

179 in a standing position with their test foot on the evaluation scale (Figure 4) (Figure 5). In this

180 position, the participants performed a lunge in which the knee was flexed in order to facilitate

181 contact between the anterior knee and a metal stick. If the patient was able to maintain contact

182 with the stick for three seconds without lifting their heel off the surface, the metal stick was

183 progressed away from the knee. As specified by developers, the "Leg Motion system test was

184 defined as the maximum distance between the toe to the metal stick where contact between the

185 stick and the knee was maintained without lifting the heel for three seconds". ${ }^{23}$

Figure 4 here

187 
191 Three trials were performed for each leg. The first was performed with one leg during three

192 seconds, and then with the other in a counterbalanced order; thus prevents muscle fatigue, by

193 alternating measurements between both legs; the mean value of the three trials was used for data

194 analysis. All the procedures were performed while the subject was barefoot. If the participants did 195 not meet any of the standards described for the test, they had to repeat the trial.

196

197 Statistical procedure

198 SPSS version 22.0 for Windows was used for statistical analysis. Descriptive statistics were 199 determined for each measurement. The mean and standard deviation (SD) were calculated for both 200 limbs. A paired t-test was performed to establish significant differences in the scores obtained at 201 test and retest sessions.

202 The intra-rater reliability was determined using ICC. The standard error of measurement (SEM) 203 and the $95 \%$ confidence intervals (CI) were calculated to estimate the error associated with the 204 measurement. ${ }^{25}$ The reliability was defined as poor (ICC $\left.<0.50\right)$, moderate (ICC 0.50 to 0.75 ), and 205 good (ICC > 0.75). ${ }^{26}$ In addition, the minimum detectable change (MDC) was calculated, based 206 on the standard error of measurement (SEM), by the formula SEM*1.96* $\sqrt{2}$, in order to avoid the 207 error range of instrument measurement. ${ }^{25}$

208 To assess the relationship between Leg Motion and other ankle dorsiflexion ROM measures, we 209 performed a Pearson correlation analysis. 


\section{RESULTS}

213 The mean values and SD were as follows: Leg Motion of the right leg was $5.15 \pm 3.08$ and on the

214 left was $5.19 \pm 2.98$; by tape measure was $5.12 \pm 3.08$ on the right and $5.12 \pm 2.80$ on the left; by 215 goniometer was $45.87^{\circ} \pm 4.98$ on the right and $44.50^{\circ} \pm 5.54$ on the left and by the inclinometer 216 app was $46.53^{\circ} \pm 4.79$ on the right and $45.27^{\circ} \pm 5.19$ on the left (Table 1 ).

Table 1 here

219 The correlation coefficients between Leg Motion and other ankle dorsiflexion ROM measurements 220 are presented in Table 2.

Table 2 here

222

223 The paired t-test showed no significant differences between the limbs and the test and re-test 224 values. The test re-test reliability results for Leg Motion were as follows: SEM ranged from 0.29 225 to $0.43 \mathrm{~cm}$, MDC ranged from 0.79 to $1.19 \mathrm{~cm}$, and ICC values ranged from 0.97 to 0.98 (Table $2263)$.

Table 3 here

228

229 DISCUSSION

230 This study showed the reliability of Leg Motion to measure ankle dorsiflexion ROM in older 231 adults. Highly reliable results were shown for the test-retest measures because SEM values ranged 232 from 0.29 to $0.43 \mathrm{~cm}$ and ICC ranged from 0.97 to 0.98 . Furthermore, the validity was stablished 233 because MDC ranged from 0.79 to $1.19 \mathrm{~cm}$. Nevertheless, it is valid due to the high correlation 234 with the established methods both the MDC and the absolute value. Therefore, its reliability was 
235 confirmed, so the Leg Motion could be an alternative choice to the classic weight-bearing test for

236 the measurement of ankle dorsiflexion ROM in elderly people. The advantage of using the Leg

237 Motion over the tape measure using the distance-to wall technique is that the subject does not have

238 to move the foot, so there aren't variations in the subtalar and foot position, or changes of the foot

239 alignment during the performance of the test which could change dorsiflexion results ${ }^{18}$.

240 In the literature, only one study was found that used this tool to evaluate university students, and

241 the authors had similar ICC values (right side 0.98; left side 0.96) for the intra-rater reliability

242 using the Leg Motion system. ${ }^{23}$ These findings support the authors' hypothesis that Leg Motion is

243 a reliable and valid tool for measuring the ankle dorsiflexion ROM in elderly adults. Moreover,

244 Calatayud et al. ${ }^{23}$ found statistically significant Pearson's coefficients $(p<0.01)$ between the Leg

245 Motion test and a tape measure, goniometer and inclinometer. This study had similar results $(\mathrm{p}<$

2460.01 ) for the tape measure and goniometer in both limbs in this specific population.

247 On the other hand, with respect to the inclinometer app results, the authors found significant 248 Pearson's correlation coefficients on the left side (ranged from 0.49 to 0.50 ), but not on the right 249 side (ranged from 0.00 to 0.02 ). In contrast to this study, Vohralik et al. ${ }^{20}$ examined the iHandy 250 Level app for the ankle dorsiflexion ROM using a weight-bearing lunge test. The test showed 251 excellent intra-rater reliability (ICC values ranged from 0.76 to 0.97 ), indicating that this app is 252 reliable and valid for measuring ankle dorsiflexion ROM.

253 Wellmont et al. ${ }^{21}$ suggest that there are errors inherent to measurement with smartphone apps due 254 to patient factors, app domain and examiner skills. In this study, the muscle fatigue, the loss of 255 balance and an altered biomechanic by age can also be predisposing factors, which may explain 256 the absence of significant Pearson's correlation coefficients on right side for the inclinometer app. 
257 The SEM and MDC results in the current study were greater than the values provided by Calatayud

258 et al. ${ }^{23}$ (intra-rater SEM ranging from 0.58 to $0.80 \mathrm{~cm}$; intra-rater MDC ranging from 1.60 to 2.23

$259 \mathrm{~cm})$ for Leg Motion measurements.

260

261 A decreased ankle dorsiflexion ROM increases instability, ${ }^{27}$ which directly impacts the loss of 262 balance and increases the fall risk in older adults. ${ }^{28}$ Therefore, it is necessary to help the elderly 263 maintain their ability to walk, climb stairs and control their gait. The number of falls is increasing 264 worldwide in older adults. ${ }^{29}$ Therefore, it is necessary to establish prevention programs to reduce 265 the fall risk.

266 Leg Motion could be very useful for monitoring the ankle dorsiflexion ROM values and as a 267 training and prevention tool. Moreover, in the study conducted by Calatayud et al., ${ }^{23}$ the metal 268 stick can be a visual target to maintain foot and knee alignment and facilitate more tactile stimulus 269 for the patient, in order to improve the right dorsiflexion execution. On the other hand, our study 270 subjects had no disturbances of balance, although it is known that old age carries a lower engine 271 and progressive loss of balance control. Maybe we could have controlled this possible fear of 272 falling, placing the Leg Motion in parallel bars, for example. We consider this aspect a limitation 273 of the study that is easily solvable for future researches.

274 Another limitation of this study is that healthy participants were studied; therefore, the results 275 cannot be extrapolated to other altered populations. Additionally, an important limitation is the 276 appearance of fatigue after standing without rest for a few minutes. Further studies are necessary 277 to improve knowledge about this device in older adults with different conditions. 
280 CONCLUSIONS

281 The Leg Motion system gives a visual and tactile stimulus that allows an adequate execution of

282 the dorsiflexion measurement, avoiding variations in the foot position. The results of the present

283 study indicated that the Leg Motion device is a valid and reliable tool that can be used as an

284 alternative to the classic weight-bearing lunge test for measuring ankle dorsiflexion ROM in older 285 adults.

286

287 ACKNOWLEDGEMENTS

288 We thank the patients who participate in this research.

289

290 REFERENCES

291 1. Skarabot J, Beardsley Ch, Hons MA, Stim I. Comparing the effects of self-myofascial 292 release with static stretching on ankle range-of-motion in adolescent athletes. Int J Sports Phys 293 Ther. 2015;10(2):203-212.

294 2. Sacco G, Turpin JM, Marteu A, Sakarovich C, Teboul B, Boscher L, Brocker P, Robert P, 295 Guerin O. Inertial sensors as measurement tolos of elbow range of motion in gerontology. Clin 296 Interv Aging. 2015;10:491-7.

297 3. Youdas JW, McLean TJ, Krause DA, Hollman JH. Changes in active ankle dorsiflexion 298 range of motion after acute inversión ankle sprain. J Sport Rehabil. 2009; 18(3):358-74.

299 4. Young R, Nix S, Wholohan A, Bradhurt R, Reed L. Interventions for increasing ankle joint 300 dorsiflexion: a systematic review and meta-analysis. J Foot Ankle Res. 2013;6(1):46.

301 5. Kolb G, Ranberg KA, Jentoft AC, O’Neill D, Topinkova E, Michel JP. Geriatric care in 302 Europe - the EUGMS Survey part I: Belgium, Czech Republic, Denmark, Germany, Ireland, 
303 Spain, Switzerland, United Kingdom. Eur Geriatric Med. 2011;2(5):290-295.

304 6. Hongo M, Itoi E, Sinaki M, Miyakoshi N, Shimada Y, Maekawa S, Okada K, Mizutani Y.

305 Effect of low-intensity back exercise on quality of life and back extensor strength in patients with

306 osteoporosis: a randomized controlled trial. Osteoporos Int. 2007; 18(10):1389-95.

307 7. Yingyongyudha A, Saengsirisuwan V, Panichaporn W, Boonsinsukh R. The Mini-Balance

308 Evaluation Systems Test (Mini-BESTest) demonstrates higher accuracy in Identifying older adult 309 participants with history of falls tan do the bestest, Berg Balance Scale, or timed up and go test. $J$ 310 Geriatr Phys Ther. 2015 (Epub Ahead of Print).

311 8. Gajdosik RL, Vander Linden DW, McNair PJ, Williams AK, Riggin TJ. Effects of an eight312 week stretching programo $\mathrm{n}$ the passive-elastic properties and function of the calf muscles of older 313 women. Clin Biomech (Bristol, Avon). 2005;20(9):973-83.

314 9. Bakker JP, de Groot IJ, Beleen A, Lankhorst GJ. Predictive factors of essation of 315 ambulation in patients with Duchenne muscular dystrophy. Am J Phys Med Rehabil. $3162002 ; 81(12): 906-12$.

317 10. Harris GF Analysis of ankle and subtalar motion during human locomotion. Inman's Joints 318 of the Ankle ed; 1991.

319 11. Pink M, Perry J, Houglum PA, Devine DJ. Lower extremity range of motion in the 320 recreational sport runner. Am J Sports Med. 1994; 22(4):541-9.

321 12. Rogan S, de Bie R, Douwe de Bruin E. Sensor-based foot-mounted wearable system and 322 pressure sensitive gait analysis : Agreement in frail elderly people in long-term care. Z Gerontol 323 Geriat. 2016; 1-10. 
324 13. Yorozu A, Moriguchi T, Takahashi M. Improved Leg Tracking Considering Gait Phase

325 and Spline-Based Interpolation during Turning Motion in Walk Tests. Sensors. 2015; 15: 2245132622472.

327 14. Venturini C, Ituassú N, Teixeira L, Deus C. Intrarater and interrater reliability of two 328 methods for measuring the actvie range of motion for ankle dorsiflexion in healthy subjets. Rev 329 Bras Fisioter. 2006;10:407-411.

330 15. Konor M, Morton S, Eckerson J, Grindstaff T. Reliability of three measures of ankle 331 dorsiflexion range of motion. Int J Sports Phys Ther. 2012;7(3):279-87.

332 16. Bennell KL, Talbot RC, Wajswelner H, Techovanich W, Kelly DH, Hall AJ. Intra-rater 333 and inter-rater reliability of a wight-bearing lunge measure of ankle dorsiflexion. Aust J Phyiother. $334 \quad 1998 ; 44(3): 175-180$.

335 17. Bohannon RW, Tiberio D, Waters G: Motion measured from forefoot and hindfoot 336 landmarks during passive ankle dorsiflexion range of motion. J Orthop Sports Phys Ther. 1991; 337 13(1):20.

338 18. Kim PJ, Peace R, Mieras J, Thoms T, Freeman D, Page J. Interrater and intrarater reliability 339 in the measurement of ankle joint dorsiflexion is independent of examiner experience and 340 technique used. J Am Podiatrc Med Assoc. 2011;101(5):407.

341 19. Venturini C, André A, Aguilar BP, Giacomelli B. Reliability of two evaluation methods of 342 active range of motion in the ankle of healthy individuals. Acta Fisiatr. 2006;13(1):39-43.

343 20. Vohralik SL, Bowen AR, Burns J, Hiller CE, Nightingale EJ. Reliability and validity of a 344 Smartphone app to measure joint range. Am J Phys Med Rehabil. 2015;94(4):325-30. 
345 21. Wellmon RH, Gulick DT, Paterson ML, Gulick CN. Validity and reliability of two 346 goniometric mobile apps: Device, Application and Examiner Factors. J Sport Rehabil. 2015 (Epub 347 ahead of print).

348 22. Cosby NL, Hertel J. Relationships between measures of posterior talar glide and ankle 349 dorsiflexion range of motion. Ath Train Sports Health Care. 2011;3(2):76-85.

350 23. Calatayud J, Martín F, Gargallo P, García-Redondo J, Colado J, Marín P. The validity and 351 reliability of a new instrumented device for measuring ankle dorsiflexion range of motion. Int $J$ 352 Sports Phys Ther. 2015;10(2):197-201.

353 24. Hoch MC, McKeon PO. Normative range of weight-bearing lunge test performance 354 asymmetry in healthy adults. Man Ther. 2011;16(5):516-519.

355 25. Weir JP. Quantifying test-retest reliability using the intraclass correlation coefficient and 356 the SEM. J Strength Cond Res. 2005;19(1):231-240.

357 26. Portney LG, Watkins MP. Foundations of Clinical Research: Applications to Practice. $3^{\text {rd }}$ 358 ed. Upper Saddle River, NJ: Pearson Prentice Hall; 2009.

359 27. Cruz-Díaz D, Lomas Vega R, Osuna-Pérez MC, Hita-Contreras F, Martínez Amat A. 360 Effects of joint mobilization an chronic ankle instability: a randomized controled trial. Disabil 361 Rehabil. 2015; 37 (7):601-10.

362 28. Schiller JS, Kramarow EA, Day AN. Fall injury episodes among noninstitutionalized older 363 adults: United States 2001-3. Advance data from vital and heatlh statistics 2007:392.

364 29. Williams J, Kowal K, Hestekin H, O’Driscall T, Peltzer K, Yawson A, Biritwum 365 R, Maximova T, Salinas Rodríguez A, Manrique Espinoza B, Wu F, Arokiasamy P, Chatterji S. 366 Prevalence, risk factors and disability associated with fall related injury in oder adults in low-and 
367 middle-incamecountries: results from the WHO study on global AGEing and adult health (SAGE).

368 BMC Med. 2015;13:147.

369 


\section{Table 1 (on next page)}

Table 1 -Results of ankle dorsiflexion range of motion measurements 
1

2

3

\begin{tabular}{ccc}
\hline Test & Side & Mean \pm SD \\
\hline Leg Motion & Right Side & $5.15 \mathrm{~cm} \pm 3.08$ \\
& Left Side & $5.19 \mathrm{~cm} \pm 2.98$ \\
Tape Measure & Right Side & $5.12 \mathrm{~cm} \pm 3.08$ \\
& Left Side & $5.12 \mathrm{~cm} \pm 2.80$ \\
Goniometer & Right Side & $45.87^{\circ} \pm 4.98$ \\
& Left Side & $44.50^{\circ} \pm 5.54$ \\
Inclinometer app & Right Side & $46.53^{\circ} \pm 4.79$ \\
& Left Side & $45.27^{\circ} \pm 5.19$
\end{tabular}

Table 1 -Results of ankle dorsiflexion range of motion measurements 2

4

5 


\section{Table 2 (on next page)}

Table 2 - Correlation coefficients between Leg Motion results and other ankle dorsiflexion range of motion measurements 
1 Table 2 - Correlation coefficients between Leg Motion results and other ankle dorsiflexion range

2 of motion measurements

3

4

\begin{tabular}{cccc}
\hline Test & Side & Leg Motion Right & Leg Motion Left \\
\hline Tape Measure & Right Side & $0.97^{*}$ & $0.86^{*}$ \\
& Left Side & $0.87^{*}$ & $0.99^{*}$ \\
Goniometer & Right Side & $0.78^{*}$ & $0.78^{*}$ \\
& Left Side & $0.51^{*}$ & $0.65^{*}$ \\
Inclinometer app & Right Side & 0.02 & $<0.01$ \\
& Left Side & $0.50^{*}$ & $0.49^{*}$
\end{tabular}

* Significant Pearson's correlation coefficients $(\mathrm{p}<0.01)$

5

6

7

8 


\section{Table 3 (on next page)}

Table 3 - Intrarater reliability for Leg Motion results on measurement 
Table 3 - Intrarater reliability for Leg Motion results

2

3

4

\begin{tabular}{lccccc}
\hline \multicolumn{1}{c}{ Side } & Mean & SD & SEM & MDC & ICC (95\% CI) \\
\hline Right Side & $5.11 \mathrm{~cm}$ & $3.04 \mathrm{~cm}$ & $0.43 \mathrm{~cm}$ & $1.19 \mathrm{~cm}$ & $0.97(0.94 ; 0.98)$ \\
Left Side & $5.19 \mathrm{~cm}$ & $2.85 \mathrm{~cm}$ & $0.29 \mathrm{~cm}$ & $0.79 \mathrm{~cm}$ & $0.98(0.95 ; 0.99)$
\end{tabular}

5

6 Abbreviations: SD: standard deviation; SEM: standard error of measurement; MDC: minimal 7 detectable difference; ICC: intraclass correlation coeficient; $\mathrm{CI}$ : confidence intervals.

8

9

10 


\section{Figure 1}

Figure 1. Position for Tape Measure protocol.

*Note: Auto Gamma Correction was used for the image. This only affects the reviewing manuscript. See original source image if needed for review. 


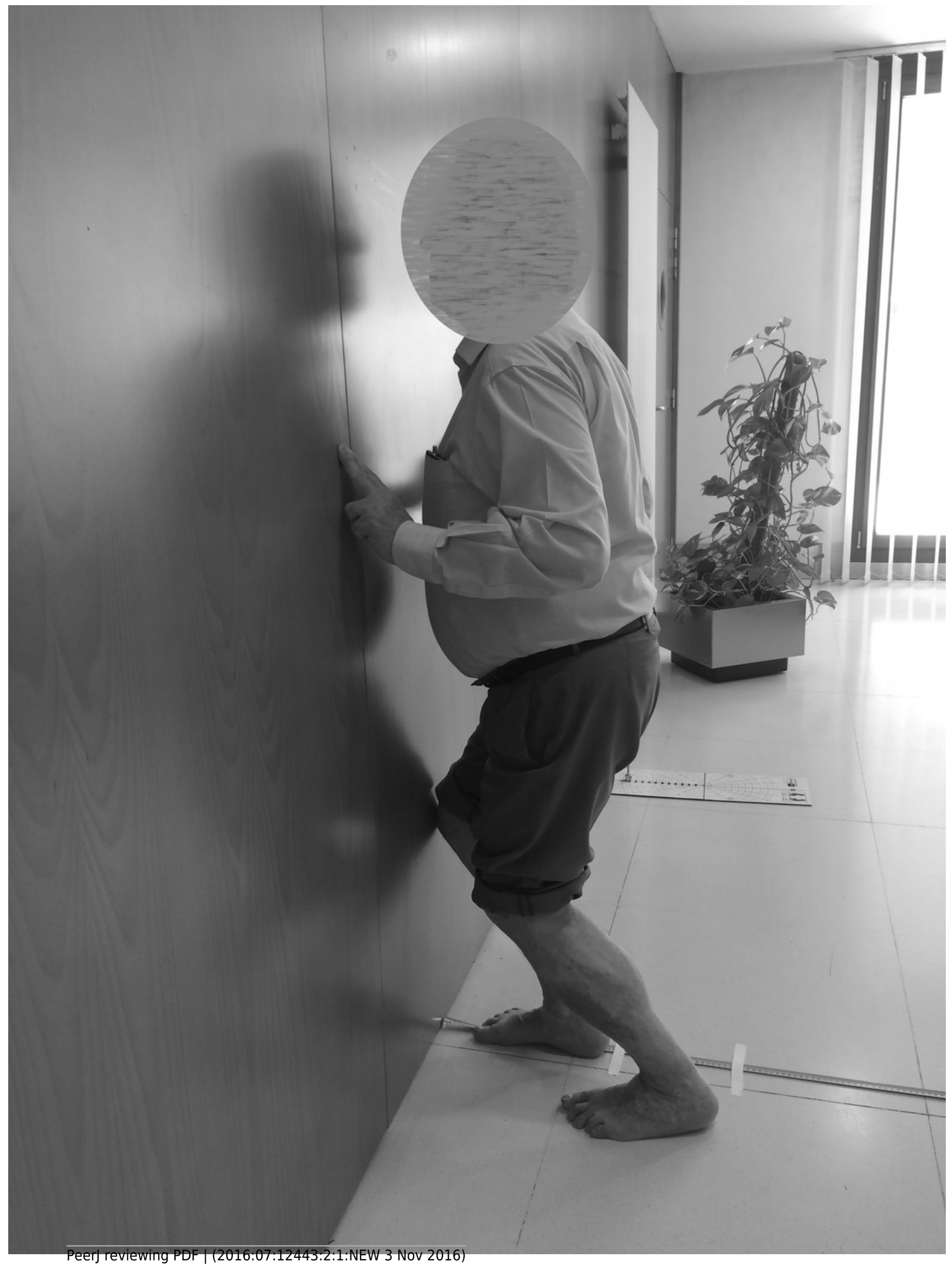




\section{Figure 2}

Figure 2. Goniometer measurement at the final lunge position in maximal dorsiflexion position.

*Note: Auto Gamma Correction was used for the image. This only affects the reviewing manuscript. See original source image if needed for review. 


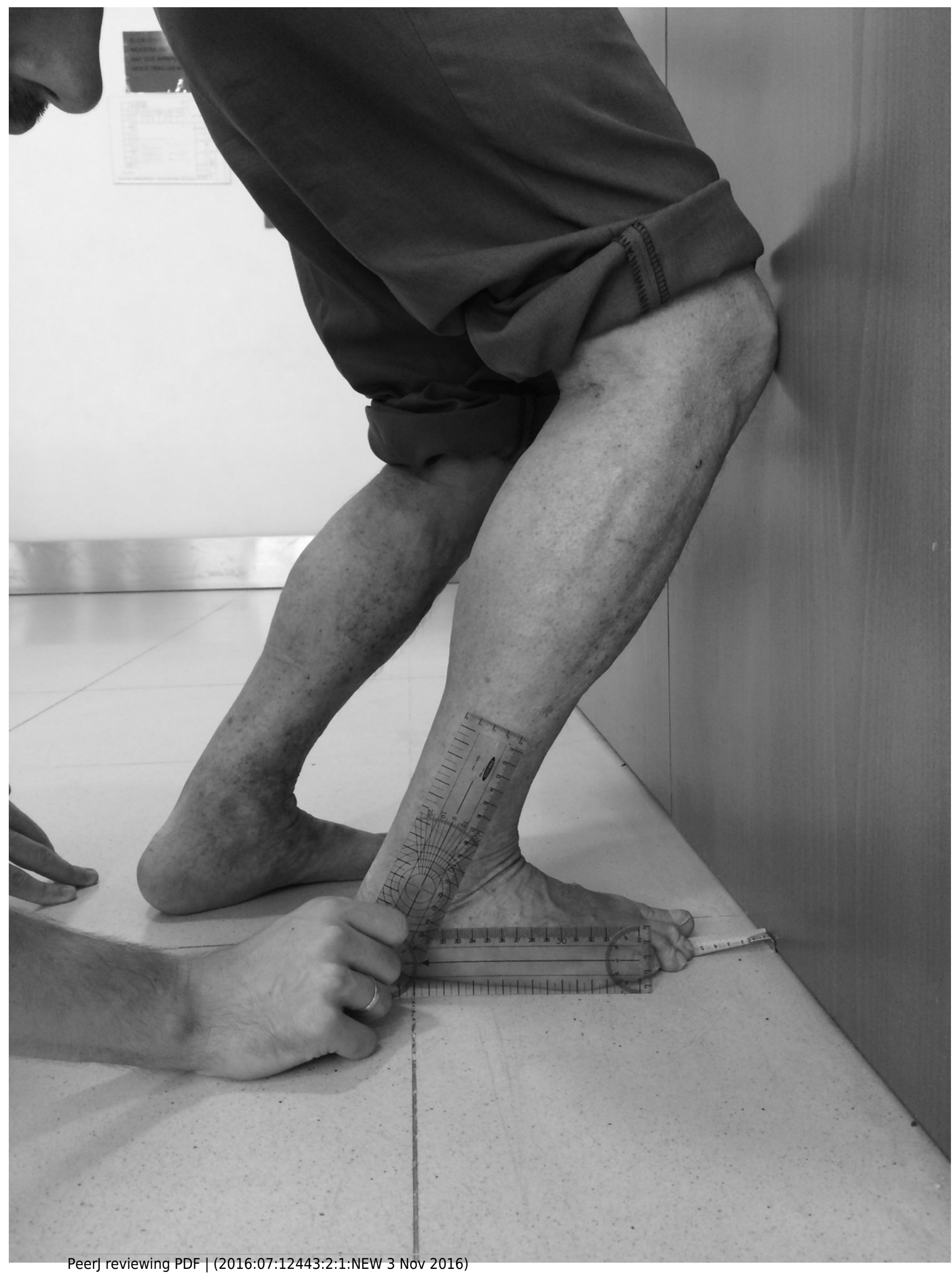




\section{Figure 3}

Figure 3. Procedure to determine the tibial tuberosity to evaluate the angle of the tibia to the ground with inclinometer app.

*Note: Auto Gamma Correction was used for the image. This only affects the reviewing manuscript. See original source image if needed for review. 


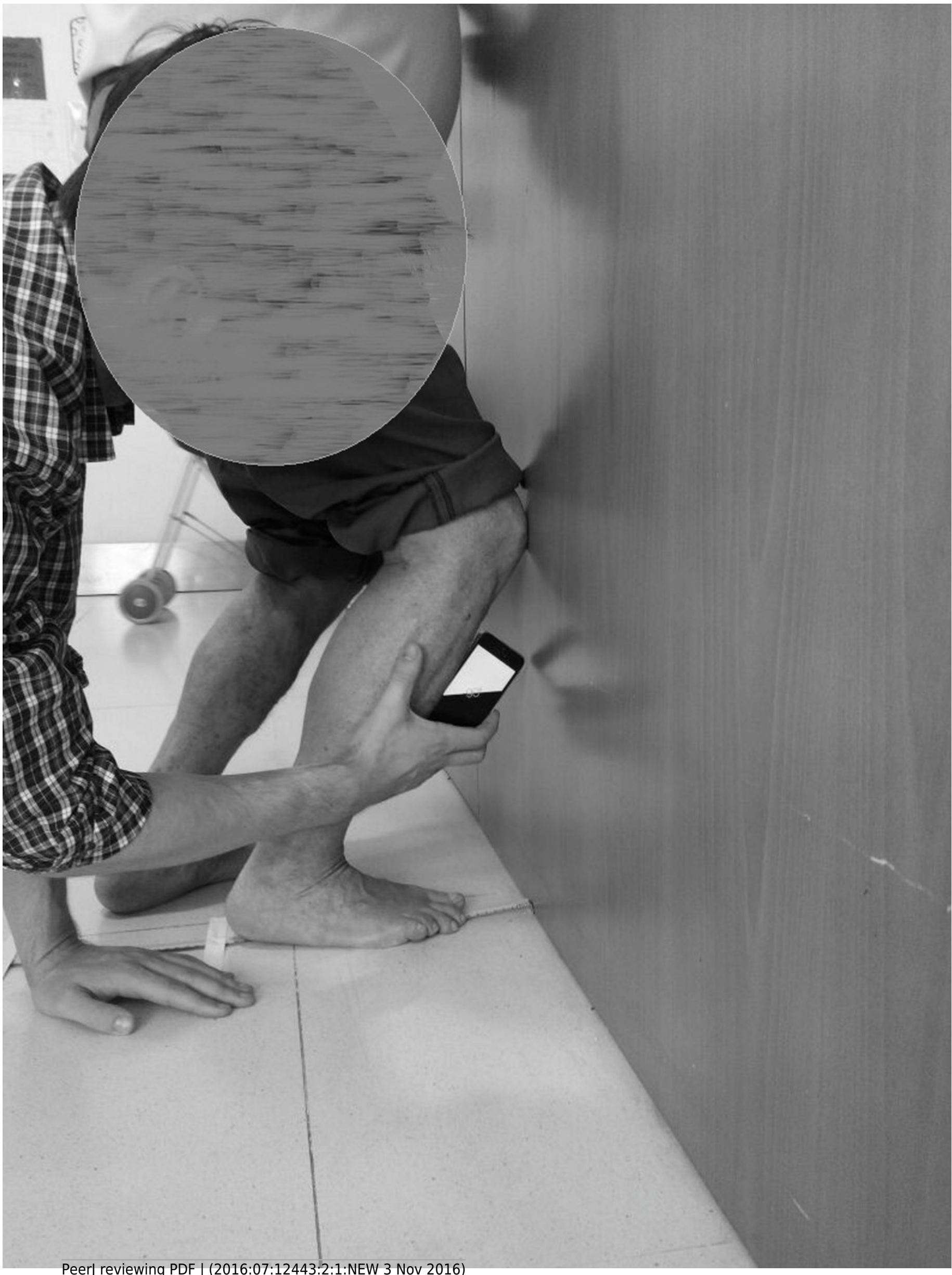




\section{Figure 4}

Figure 4. Leg Motion system and procedure.

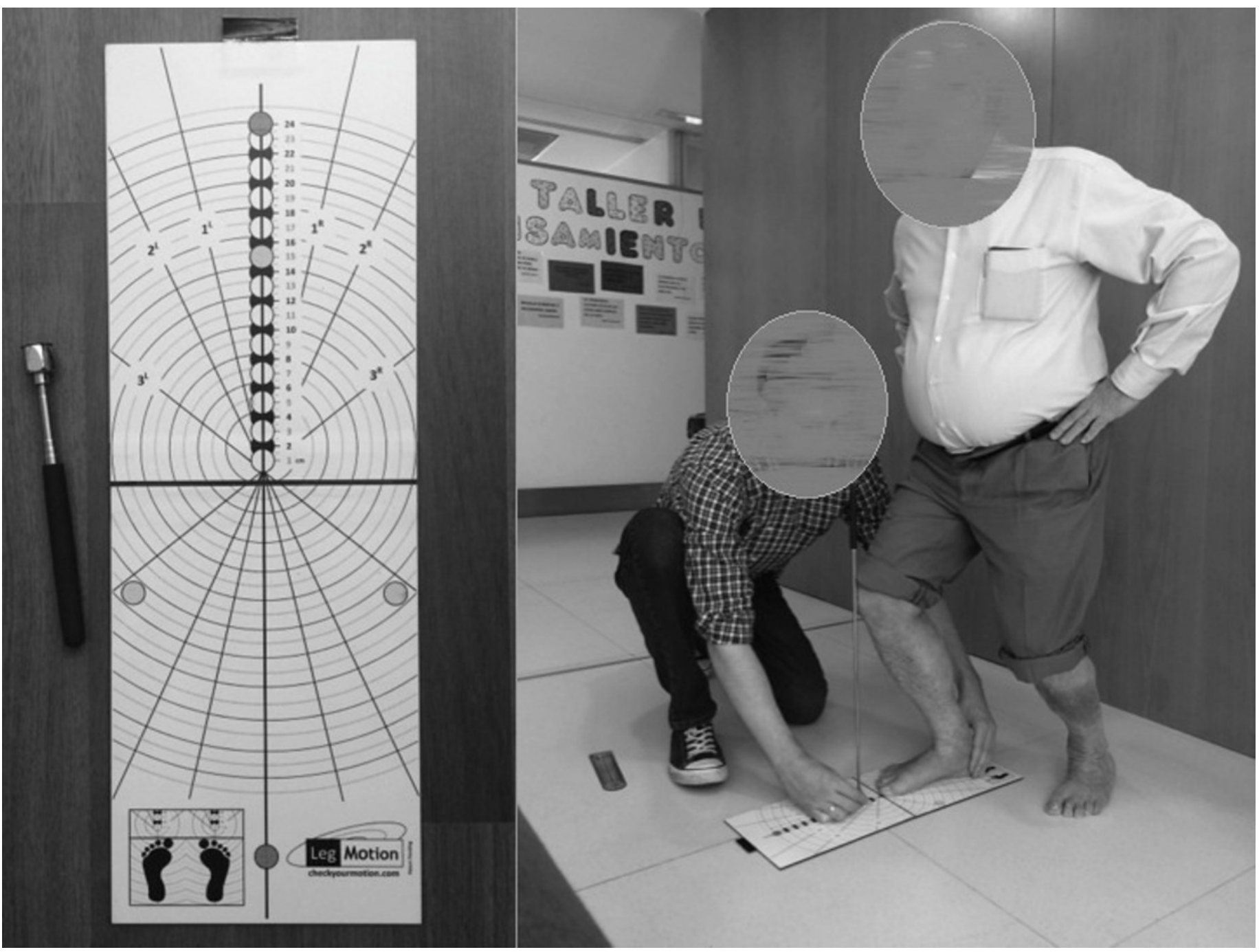




\section{Figure 5}

Figure 5. Leg Motion procedure.

*Note: Auto Gamma Correction was used for the image. This only affects the reviewing manuscript. See original source image if needed for review. 


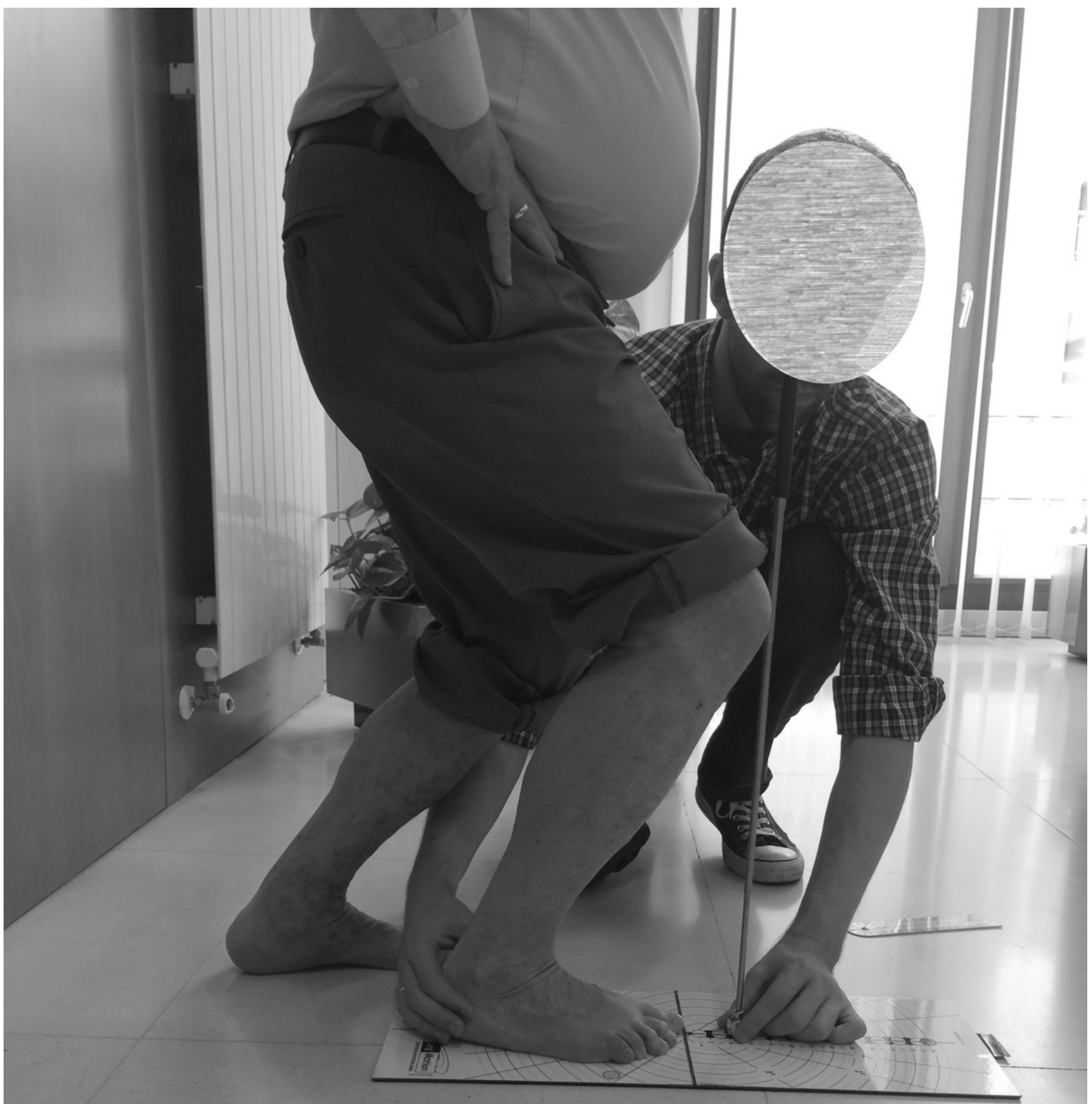

